\title{
On the analogy between the evolution of thermodynamic and bibliometric systems: a breakthrough or just a bubble?
}

\author{
Fiorenzo Franceschini ${ }^{1}$ and Domenico Maisano ${ }^{2}$ \\ ${ }^{1}$ fiorenzo.franceschini@polito.it 22domenico.maisano@polito.it \\ Politecnico di Torino, DISPEA (Department of Production Systems and Business Economics), \\ Corso Duca degli Abruzzi 24, 10129, Torino (Italy)
}

\begin{abstract}
This paper presents an in depth study of an interesting analogy, recently proposed by Prathap (Scientometrics 87(3):515-524, 2011), between the evolution of thermodynamic and bibliometric systems. The goal is to highlight some weaknesses and clarify some "dark sides" in the conceptual framework of this analogy, discussing the formal validity and practical meaning of the concepts of Energy, Exergy and Entropy in bibliometrics. Specifically, this analogy highlights the following major criticalities: (1) the definitions of $E$ and $X$ are controversial, (2) the equivalence classes of $E$ and $X$ are questionable, (3) the parallel between the evolution of thermodynamic and bibliometric systems is forced, (4) $X$ is a non-monotonic performance indicator, and (5) in bibliometrics the condition of "thermodynamic perfection" is questionable.
\end{abstract}

Argument is supported by many analytical demonstrations and practical examples.

Keywords: Bibliometrics, Thermodynamics, Exergy, Energy, Entropy, $S=E-X, p$-index, composite indicators.

\section{Introduction}

In a recent paper in Scientometrics, Prathap (2011a) introduces an interesting analogy between the evolution of a bibliometric system - interpreted as the accumulation process of the publications and corresponding citations of a scientist (or a group or any larger aggregation) - and the thermodynamic evolution of a physical system. According to Prathap, the analogy helps to "assess more meaningfully the bibliometric progress of a scientist" [Prathap, 2011a, page 522].

Precisely, if one considers the scientific production of a scientist over a given time window, it will include a total number of publications $(P)$, with $\left(c_{i}\right)$ citations each, being $i=1, \ldots, P$. Obviously, the total number of received citations is given by:

$C=\sum_{i=1}^{P} c_{i}$ 
In analogy with thermodynamic processes, Prathap (2011a) defines the concepts of energy $(E)$, exergy $(X)$ and entropy $(S)$ in bibliometrics as:

$$
E=\sum_{i=1}^{P} c_{i}^{2},
$$

which is the total available energy of the system,

$$
X=\left(\frac{C}{P}\right)^{2} \cdot P=\frac{C^{2}}{P},
$$

which represents the exergy, that is the "work potential" or "free energy" of $E$, according to the Second Law of Thermodynamics, and

$$
S=E-X,
$$

which is a measure of the unevenness or "disorder" of the publication portfolio. Indicators $E$, $X$, and $S$ - also known as EEE (Energy, Exergy, Entropy) trinity - can be used for monitoring the temporal evolution of a scientist's scientific output. For more details on the philosophy behind their definition, we refer the reader to [Prathap, 2011a; 2011b]. Among the indicators of the EEE trinity, Prathap argues that the most powerful is $X$, "a meaningful single number scalar measure of a scientist's performance" [Prathap, 2011a, page 515].

The purpose of this paper is to make explicit our reflections on the Prathap's analogy, underlining strengths and (especially) inconsistencies. There are five basic criticalities that will be analysed in detail: (1) the definition of $E$ and $X$ is controversial, (2) the equivalence classes of $E$ and $X$ are questionable, (3) the parallel between the evolution of thermodynamic and bibliometric systems is forced, (4) $X$ is a non-monotonic performance indicator, and (5) in bibliometrics the condition of "thermodynamic perfection" is questionable.

The remaining of this paper is organised into four sections. Section 2 recalls some basic notions of thermodynamics, which are useful to understand the nature of this analogy. Section 3 contains some reflections on the peculiarities of $E, X$ and $S$, when used in a bibliometric context. Section 4 contains a structured criticism on the analogy, emphasizing its limitations and contradictions under the viewpoints of the five criticalities mentioned before. Discussion is supported by analytical demonstrations and practical examples. Finally, the conclusions are given, summarising the original contribution of the paper.

\section{Basic notions of Thermodynamics}

A generic thermodynamic system has - at a given time - a total energy $(E)$, which can have many forms (chemical, electrical, mechanical, thermal, etc...). Assuming that the system of interest is isolated, i.e. no energy (heat and work) or mass can be transferred in or out of the 
system boundary, only a portion of $E$, called exergy $(X)$, can be transformed into work. The Second Law of Thermodynamics asserts that in any transfer or conversion of energy within an isolated system, the energy "quality" or "work potential" decreases because a portion of this energy is transformed in "disordered energy of the system", basically heat that can not be converted back into work [Dincer and Cengel, 2001]. Entropy $(S)$ is a thermodynamic property that is simply a measure of the amount of "disorder" within a system. $S$ of an isolated system can only increase (not be destroyed) and reach the maximum possible value, which is the state of thermodynamic equilibrium to which the system spontaneously and irreversibly evolves. On the other hand, the entropy of an open system can be increased/decreased by energy or mass transports across the system boundary. For a system in thermodynamic equilibrium, the relationship between $E, X$ and $S$ is given by:

$E=X+T_{R} \cdot S$,

where $T_{R}$ is the reference temperature of the system's surroundings. We remark that all the terms in Eq. 1 are non-negative. Only the portion $X$ of the available energy $(E)$ can be converted into work, while the remaining energy $\left(T_{R} \cdot S\right)$ is unusable or "disordered". The relationship proposed by Prathap (Eq. 4) is analogous to Eq. 5, with the only exception that term $T_{R}$ is not present.

\section{A conceptual framework}

The starting point of the thermodynamic-bibliometric consilience is the definition of $X$ (Eq. 3 ), which can be seen as the product of $P$ - indicator of global productivity - and the square of $C / P$, i.e. the average citations per publication - indicator of efficiency in terms of average impact/diffusion. According to the Glänzel-Schubert model (Eq. 6), $X$ is proportional to the well known $h$-index [Hirsch, 2005; Glänzel, 2006; Schubert and Glänzel, 2007; Ye, 2011]:

$h \sim P^{1 / 3}(C / P)^{2 / 3}=\left(C^{2} / P\right)^{1 / 3}=X^{1 / 3}$.

Prathap argues that $X$ or its "next-of-kin" $p=X^{1 / 3}$, also known as mock $h$-index, may substitute or even improve $h$ (“...there may well be merit in treating this (i.e. $p$ ) as a substitute or mock $h . . . "$ [Prathap, 2010, page 155]; “...X is a more meaningful, if not more accurate, single number scalar measure of a scientist's performance. Neither $P$ nor $C$, nor $C / P$, nor even the popular $h$-index, serves this purpose" [Prathap, 2011a, page 523]).

The energy $\left(e_{i}\right)$ of a single $i$-th publication with $c_{i}$ citations is defined as:

$e_{i}=\frac{c_{i}^{2}}{1}=c_{i}^{2}$. 
Thus, $e_{i}$ can be seen as the exergy that this publication would entail, if it represented the entire scientific production (Eq. 7 is obtained by applying Eq. 3 with $C=c_{i}$ and $P=1$ ). $E$ is then given by the sum of the energies ( $\left.e_{i}\right)$ of the single publications (Eq. 2).

Without worrying (for the moment) about the meaningfulness of the EEE indicators' definition, it is interesting to note that $X \leq E$, consistently with thermodynamics. This can be shown as follows:

$$
S=E-X=\sum_{i=1}^{P} c_{i}^{2}-\bar{c}^{2} \cdot P=\sum_{i=1}^{P} c_{i}^{2}-\sum_{i=1}^{P} \bar{c}^{2}=\sum_{i=1}^{P}\left(c_{i}^{2}-\bar{c}^{2}\right),
$$

being $\bar{C}=C / P$, i.e. the average citations per publication. Next, Eq. 8 can be turned into this form:

$$
\begin{aligned}
S & =\sum_{i=1}^{P}\left[\left(c_{i}^{2}+\bar{c}^{2}-2 \cdot c_{i} \cdot \bar{c}\right)+2 \cdot c_{i} \cdot \bar{c}-2 \cdot \bar{c}^{2}\right]=\sum_{i=1}^{P}\left[\left(c_{i}-\bar{c}\right)^{2}+2 \cdot c_{i} \cdot \bar{c}-2 \cdot \bar{c}^{2}\right]= \\
& =\sum_{i=1}^{P}\left[\left(c_{i}-\bar{c}\right)^{2}+2 \cdot \bar{c}\left(c_{i}-\bar{c}\right)\right]=\sum_{i=1}^{P}\left(c_{i}-\bar{c}\right)^{2}+2 \cdot \bar{c} \cdot \sum_{i=1}^{P}\left(c_{i}-\bar{c}\right) .
\end{aligned}
$$

In the second member of Eq. 9, the quantity $\sum_{i=1}^{P}\left(c_{i}-\bar{c}\right)$ is null by definition, since it is the sum of the deviations of the $c_{i}$ values about their mean $(\bar{c})$. Therefore, Eq. 9 will turn into:

$$
S=\sum_{i=1}^{P}\left(c_{i}-\bar{c}\right)^{2}
$$

which is always $\geq 0$. Since the three EEE indicators are non-negative quantities, it follows that $0 \leq X \leq E$. Alternatively, this condition can be proved using the inequality of CauchySchwarz.

Being given by the summation of (squared) integer values (see Eq. 2), $E$ is defined over the domain of natural numbers $\mathbf{N}_{0}$. On the other hand, since $\bar{c}$ can be a fractional number, $S$ and $X$ are defined over the domain of real positive numbers $\mathfrak{R}_{0}^{+}$.

The maximum possible value of $X$ is $E$, when $c_{i}=\bar{C}$ for each $i=1$ to $P$, that is, all the publications have exactly the same number of citations. According to Prathap (2011a), this is the "thermodynamically perfect" case, because $S=0$ and $X=E$. The opposite situation is when the whole $E$ is "not usable to do work" $(X=0)$. Let us show when this condition is met. Eq. 4 can be expressed as:

$$
S=E-\bar{c}^{2} \cdot P=\sum_{i=1}^{P} c_{i}^{2}-\bar{c}^{2} \cdot P
$$


The only case in which $S=E$ is when the non-positive term $-\bar{c}^{2} \cdot P=0$. Since $c_{i}$ values cannot be negative, this entails that $c_{i}=0$ for each $i=1$ to $P$ and therefore EEE indicators "degenerate" into $E=X=S=0$. What is the meaning of this situation from a bibliometric viewpoint?

Going on with the exam of this analogy, it can be noticed that the bibliometric EEE indicators are state functions, since their value do not depend on the (thermodynamic) path that the system took to get between two "equilibrium states", but depends only on the current state of the system, which is described by a set of variables. While in thermodynamics, variables are pressure, temperature, magnetic field, mass, etc..., those defining a bibliometric state should be the number of publications and the relevant citations $\left(c_{i}\right)$. It is trivial to show that, given a final bibliometric state, the order in which publications or citations have been accumulated over time has no effect on the values of the EEE indicators. Since $E$ and $S$ are expressed as sum of terms relating to the citations of each publication (see Eqs. 2 and 10), the final result is not influenced by the order of addends (commutative property of addition). For the purpose of example, Table 1 shows the progress of a fictitious scientist's output along two alternative paths ( $P 1$ and $P 2)$, which bring to the same final "bibliometric state" (instant $t_{3}$ ). In the instant $t_{3}$ there are three total publications, with a certain number of citations each. Dash "-" indicates that the publication of interest has not yet been issued. For instance, considering $P 1$, in $t_{2}$ there are two publications only (i.e. the $1^{\text {st }}$ and the $2^{\text {nd }}$ ), with 1 and 2 citations respectively. The corresponding data can be graphically represented by a specific diagram proposed by Prathap (in Fig. 1). The values of EEE indicators at instant $t_{3}$ are not influenced by the bibliometric path to reach the final state, but only depend on the citation statistics of that state.

Table 1. Chronological sequence of the publication output of a fictitious scientist. The publications and corresponding citations at instant $t_{3}$ can be accumulated following two alternative "paths" (P1 and P2). For simplicity, three total publications are considered, reporting their citation accumulation $\left(c_{i}\right.$ values) over time. Dash “-” indicates that, in a particular time instant, the publication of interest has not yet been issued. For each of the time instants $\left(t_{i}\right)$ considered, the following indicators are also reported: $P, C, h$, and the three EEE indicators (i.e. $E, X$ and $S$ ).

\begin{tabular}{rlrrrrrrrrr}
\hline Path & Instant & $c_{1}$ & $c_{2}$ & $c_{3}$ & $P$ & $C$ & $h$ & $E$ & $X$ & $S$ \\
\hline \multirow{3}{*}{$P 1$} & $t_{1}$ & 1 & - & - & 1 & 1 & 1 & 1 & 1.0 & 0.0 \\
& $t_{2}$ & 1 & 2 & - & 2 & 3 & 1 & 5 & 4.5 & 0.5 \\
& $t_{3}$ & 1 & 2 & 3 & 3 & 6 & 2 & 14 & 12.0 & 2.0 \\
\hline \multirow{3}{*}{$P 2$} & $t_{1}$ & - & - & 3 & 1 & 3 & 1 & 9 & 9.0 & 0.0 \\
& $t_{2}$ & - & 2 & 3 & 2 & 5 & 2 & 13 & 12.5 & 0.5 \\
& $t_{3}$ & 1 & 2 & 3 & 3 & 6 & 2 & 14 & 12.0 & 2.0 \\
\hline
\end{tabular}




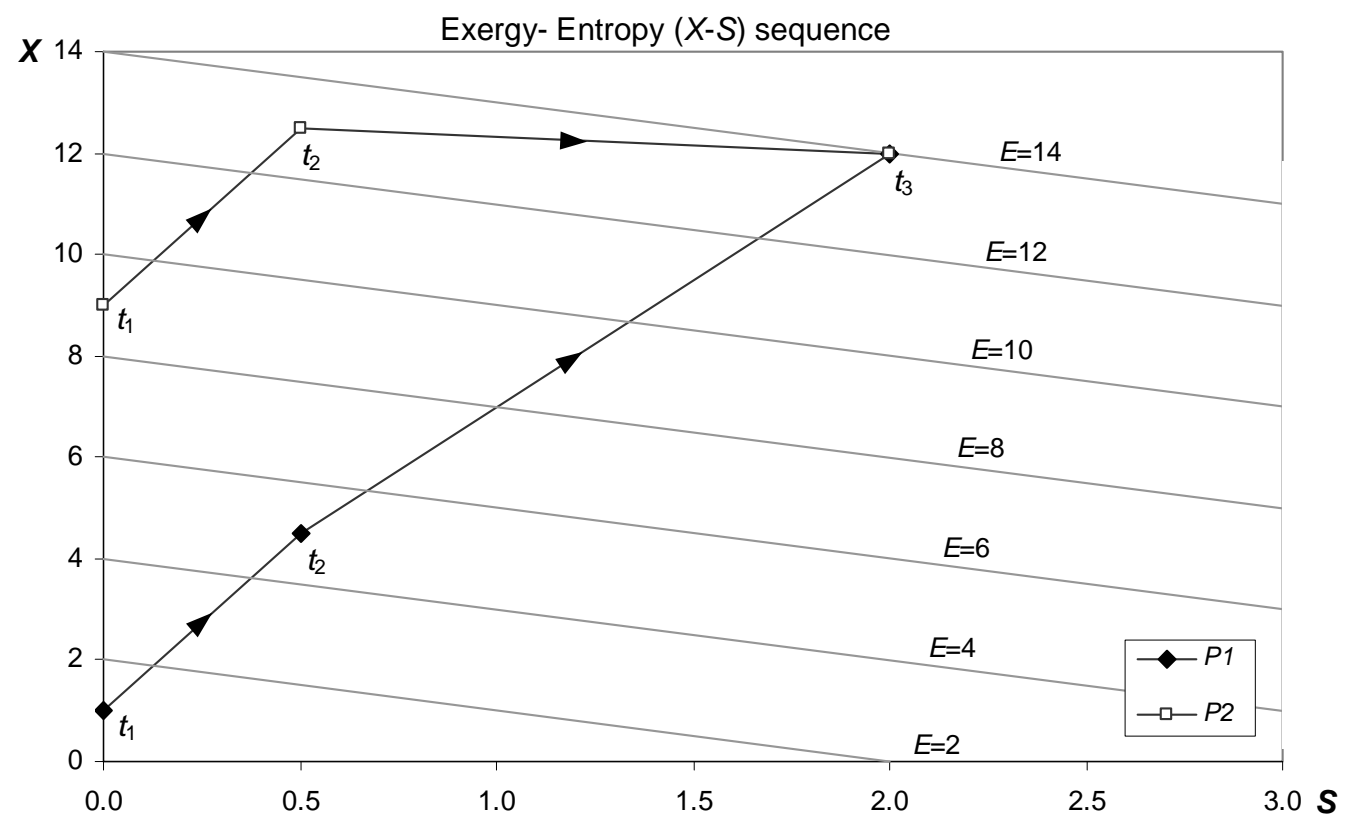

Fig. 1. Energy-Exergy-Entropy diagram, representing the chronological $X$ - $S$ sequence of the output of a fictitious scientist, along two alternative "paths" (P1 and P2). Numerical data are reported in Table 1. Iso- $E$ curves appear in this diagram as parallel solid lines.

\section{Criticism on the analogy}

Despite the similarities between the evolution of thermodynamic and bibliometric systems, which are emphasised by Prathap (2011a) and partly recalled in Sect. 3, many ambiguities and inconsistencies emerge. They are illustrated individually in the following sub-sections.

\subsection{Controversial definition of $X$ and $E$}

Let us examine again the definition of $X$ (Eq. 3). While it is not unreasonable to assert that $P$ and $C / P$ are rough indicators, respectively of quantity - in terms of global production - and quality - in terms of average impact/diffusion - on the other hand, it cannot be said that $X$ synthesises effectively the two dimensions of interest. In fact, the risk of this and other composite indicators is "crashing" dimensions by a dubious aggregation criterion [Glänzel, 2006; Franceschini and Maisano, 2007; 2011a]. Obviously the aggregation criterion proposed by Prathap, derives from the fact that the quantity $C^{2} / P$ is likely to be proportional to the $h$ index (see Eq. 6), but we should not forget that:

- While not discussing the terms $C / P$ and $P$ - which, actually, are not very robust since they can be influenced by "big-hits" and low-cited publications [Franceschini and Maisano, 2011c] - we do not think that their combination by the product leads to obtain an indicator $(X)$, which "overcomes the many deficiencies of the $h$-index" [Prathap, 2011a, page 156]. 
Instead, this aggregation undermines its simplicity and immediacy [Franceschini and Maisano, 2010].

- Prathap deliberately ignores the fact that the relationship in Eq. 6 fits best on aggregated levels (i.e. with thousands of publications, such as for institutions, journals, countries, and so on), than applied to a single scientist. In the latter case there can be huge deviations from the theoretical (Glänzel-Schubert's) model [Ye, 2011].

\subsection{On the meaning of $S$ and $E$}

$S$ is depicted as a "measure of the unevenness (disorder) of the publication portfolio" [Prathap, 2011a, 515]. This is correct, but the point is probably much simpler. Eq. 10 shows that $S$ is nothing else than the sum of the squared deviations of $c_{i}$ values, which is a variance multiplied by $(P-1)$, often abbreviated as SS in statistics. Following this interpretation, the quantity $S /(P-1)$ is exactly the variance $\left(\sigma^{2}\right)$ of the $c_{i}$ values. As a consequence, $E$ is the sum of $X$ - which is an indicator of performance, "next-of-kin" of $h-$ and $S$ - which is the SS of the $c_{i}$ values. How can we say that $E$ represents the available energy, of which the fraction $X$ is the "work potential"? In our opinion, $E$ is an indicator with no practical meaning, because it is obtained by summing apples ( $X$, indicator of performance) and oranges ( $S$, indicator of dispersion).

\subsection{Iso-energetic and iso-exergetic states}

Our puzzlement on the practical meaning of $E$ may become more comprehensible when examining iso-energetic bibliometric states - that is to say states with the same E. Let us consider an example: Table 2 reports the citation statistics of six fictitious scientists (S1 to $S 6) . S 1$ to $S 4$ have the same energy $(E=25)$, therefore, in the diagram in Fig. 2, they lay on the same iso-energetic line. The $c_{i}$ values of $S 1$ and $S 3$ are more dispersed than those of $S 2$ and $S 4$, as also denoted by the relatively higher $S$ values. From the energetic point of view, the first four scientists have the same $E$; is this equivalence still valid in terms of bibliometric performance? In other words, are those four scientists bibliometrically equivalent? Probably not, considering their difference in terms of $P, C, h$, as well as in terms of $X$ - i.e. the performance indicator suggested by Prathap. Unsurprisingly, it can be noticed a partial disagreement between the values of $X$ and $h$, both indicators of a scientist's performance, as a (pleonastic) proof of the fact that their link is not respected for small sets of publications.

While the practical interpretation of iso-energetic states is quite dubious, what can we say about the iso-exergetic ones? Let try to answer this question by further examining the 
example of Table 2. S2 and $S 4$ are not only iso-energetic, but also iso-exergetic (i.e. same $X=25$ ). According to Prathap's words, since $S 2$ and $S 4$ have the same $E$ and $X$, they also have the same performance and "technical working capacity" [Prathap, 2011a, page 519]. Can we now affirm that these pairs of scientists are bibliometrically equivalent? One can also notice that $S 6$ and $S 7$ are iso-exergetic but they have different $E$ values. Even assuming that they are equivalent in terms of performance, is it reasonable to say that $S 6$ is more efficient than $S 7$, because his/her amount of "disordered energy" is smaller? Another dubious comparison is that between S4 and S5. S5 has a higher "technical working capacity" $(X)$ but is also more "disordered" (higher $S$ ). How can we evaluate these scientists and measure their difference by their $X$-S values.

Table 2. Citation statistics relating to six fictitious scientists ( $S 1$ to $S 6$ ). For each scientist, the following indicators are reported: $P, C, C / P, h$, and the three EEE indicators (i.e. $E, X$ and $S$ ).

\begin{tabular}{clrrrrrrr}
\hline \multicolumn{2}{l}{ Scientist Citation statistics } & $P$ & $C$ & $C / P$ & $h$ & $E$ & \multicolumn{1}{c}{$X$} & \multicolumn{1}{c}{$S$} \\
\hline$S 1$ & $c_{1}=3 \quad c_{2}=4$ & 2 & 7 & 3.50 & 2 & 25 & 24.50 & 0.50 \\
$S 2$ & $c_{1}=5$ & 1 & 5 & 5.00 & 1 & 25 & 25.00 & 0.00 \\
$S 3$ & $c_{1}=1 \quad c_{2}=2 \quad c_{3}=2 \quad c_{4}=4$ & 4 & 9 & 2.25 & 2 & 25 & 20.25 & 4.75 \\
$S 4$ & $c_{i}=1$ for each $i=1$ to 25 & 25 & 25 & 1.00 & 1 & 25 & 25.00 & 0.00 \\
$S 5$ & $c_{i}=6$ for $i=1$ and $c_{i}=1$ for each $i=2$ to 25 & 25 & 30 & 1.20 & 1 & 60 & 36.00 & 24.00 \\
$S 6$ & $c_{i}=0$ for each $i=1$ to 16 and $c_{i}=4$ for each $i=17$ to 32 & 32 & 64 & 2.00 & 4 & 256 & 128.00 & 128.00 \\
$S 7$ & $c_{i}=1$ for each $i=1$ to $10, c_{i}=3$ for each $i=11$ to 16 and $c_{i}=10$ for & & & & & & \\
& each $i=17$ to 18
\end{tabular}

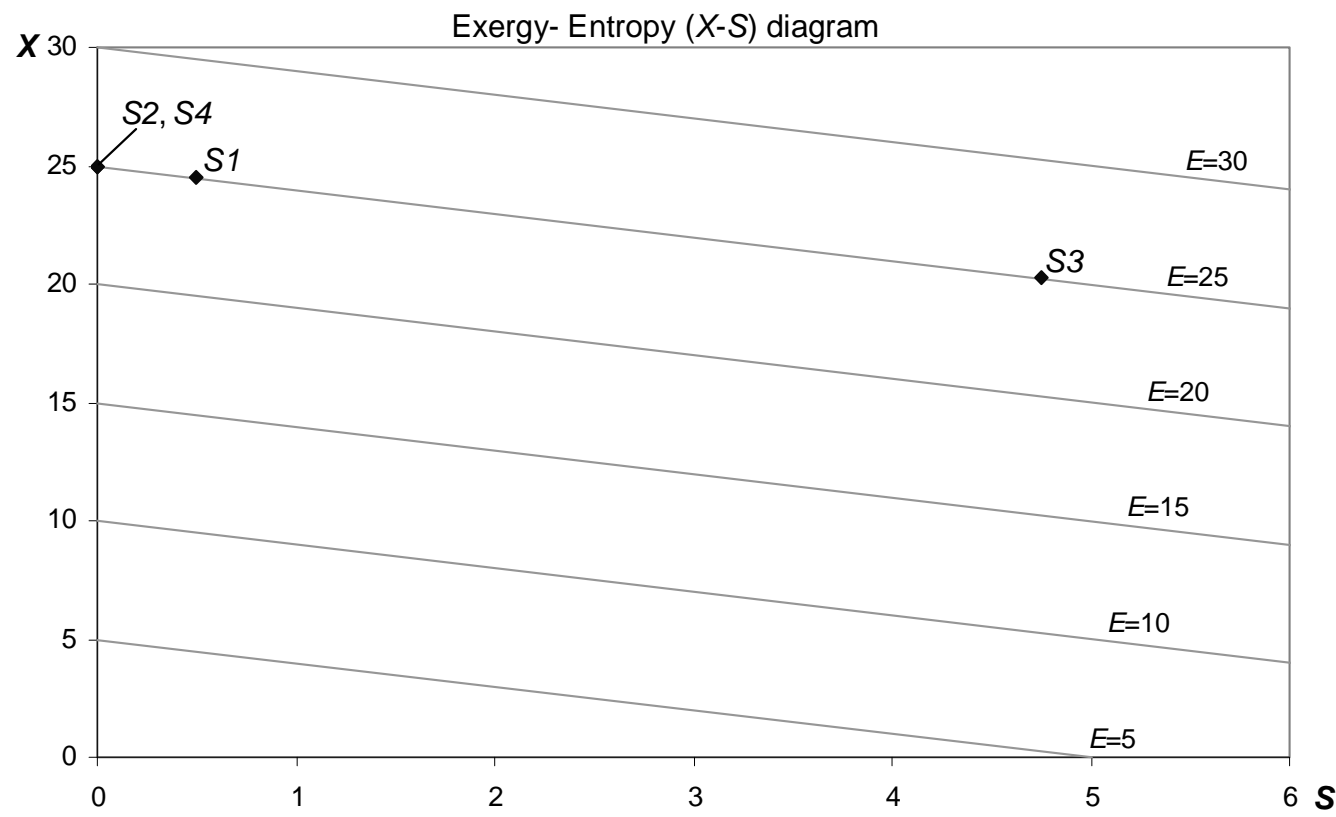

Fig. 2. Energy-Exergy-Entropy diagram, representing the $X-S$ positioning of the overall output of five fictitious scientists (S1 to S4). Numerical data are reported in Table 2. 
Let deepen our analysis by introducing a new representation. Fig. 3 is a $C-P$ plan for representing the bibliometric positioning of a scientist, according to his/her $P$ and $C$ values. Iso- $X$ curves $\left(C^{2} / P=\right.$ constant $)$, that is the geometric loci of the points with equal performance in terms of $X$, appear as some arcs of a parabola with horizontal axis. Now the question is: what is the rationale for considering two points laying on the same arc of parabola as bibliometrically equivalent? For example, $S 6$ and $S 7$ have coincident $X$ indices $(X=128)$, even if $S 6$ is superior than $S 7$, regarding both $P$ and $C$ values, but his/her $C / P$ ratio is smaller (2 against 2.7). Similar considerations can be extended to $S 2$ and $S 4$. The synthesis by $X$ introduces a questionable equivalence that subverts the classifications according to $C, P, C / P$.

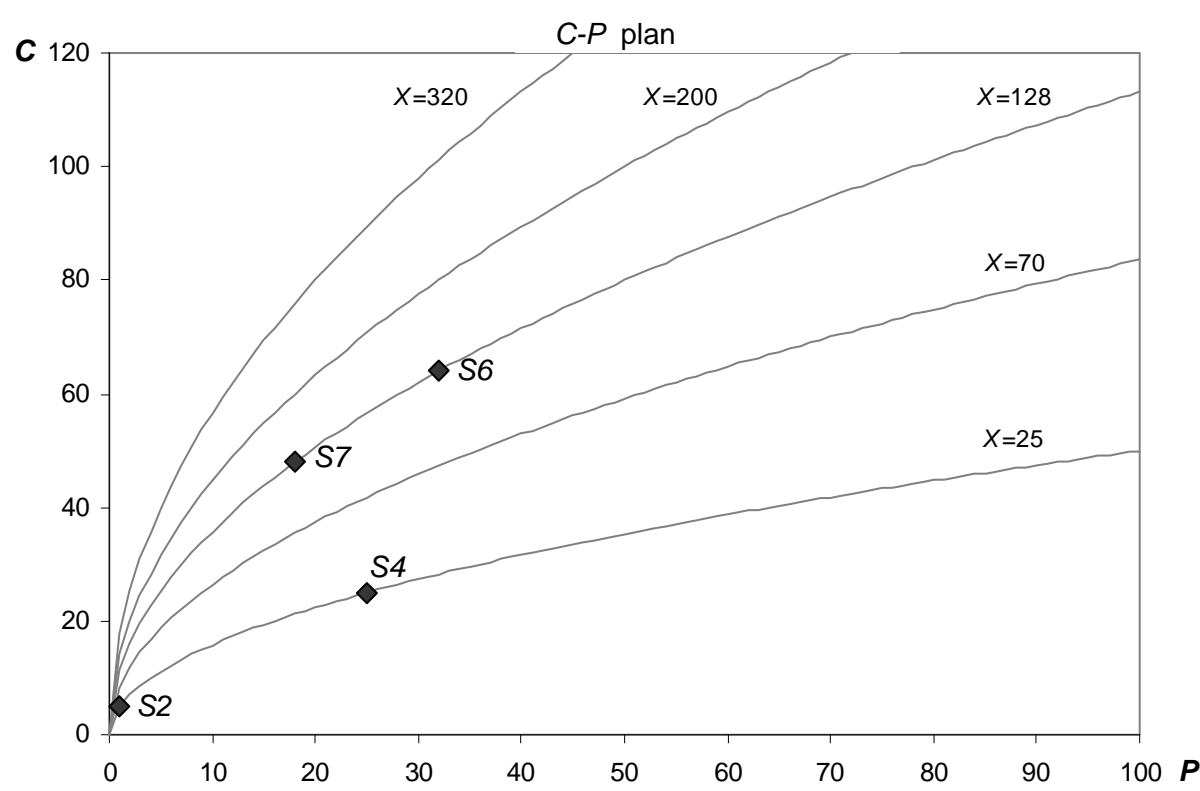

Fig. 3. $C$ - $P$ plan representing the bibliometric positioning of scientists. Iso- $X$ curves appear as some arcs of a parabola with horizontal axis. $S 2-S 4$ and $S 6-S 7$ are two pairs of scientists with coincident $X$ values (respectively 25 for $S 2-S 4$ and 128 for $S 6-S 7$ ) but different $P$ and $C$ values (see Table 2). Are they equivalent in terms of bibliometric performance or not?

Regarding $X$, it can be also noticed that the substitution rate - defined as the rate at which the $P$ can be increased/decreased in exchange for a decrease/increase in $C$, maintaining the same $X$ value - is not constant. Assuming that $P$ and $C$ are continuous variables, the substitution rate would be:

$$
\left(\frac{C^{2}}{P}=\text { constant }\right) \rightarrow \frac{\Delta C}{\Delta P}=\frac{1}{2} \sqrt{\frac{\text { constant }}{P}}=\frac{1}{2} \cdot \frac{C}{P}=\frac{\bar{c}}{2},
$$

which can be geometrically represented by the angular coefficient of the line tangent to the iso- $X$ curve. Eq. 12 says that this quantity is not constant over the $P$ domain, since it depends on the scientist's $P$ and $C$ values. For example, considering the previous example, substitution rate of $S 6$ is exactly 1 , while that one of $S 7$ is $\approx 1.33$. In other words, the growth in terms of 
citations/publications, so as to counterbalance a certain increase in terms of publications/citations while maintaining the same $X$, depends on the "operating point". What is the rationale behind?

\subsection{Thermodynamic evolution of a bibliometric system: does it make sense?}

Simplifying, the Second Law of Thermodynamics says that in an isolated thermodynamic system, each spontaneous transformation process is irreversible $(\Delta S>0)$ [Dincer and Cengel, 2001]. Ideal transformations - which can never really happen - are characterized by $\Delta S=0$, because they do not generate "disordered energy", that is, heat that cannot be turned into work. If the system is not isolated, that is it can exchange energy and matter with the exterior, then its $S$ may also decrease. An air conditioner, for example, may cool the air in a room, thus reducing the entropy of the air of that (non-isolated) system [Dincer and Cengel, 2001].

Now let us explore whether the previous concepts are also valid for bibliometric systems. We think that the ensemble of scientific publications and citations of a scientist can be seen as a non-isolated system. In fact, according to Eq. 2, every new citation (relating to a publication already in the portfolio or to a new publication) will make $E$ increase. Thus, the accumulation of citations is a kind of energy supply to the system from the "surroundings". After this transfer of energy, the system immediately reaches a new state of equilibrium and the EEE indicators will change as follows:

- $E$ will only increase (see Eq. 2);

- $X$ will increase or decrease (demonstration is given later);

- $S$ will increase or decrease (demonstration is given later)

These changes are visible in the example reported in Table 3, concerning the chronological sequence of the publication output of a fictitious scientist. The $c_{i}$ values associated to the publications are reported in the columns on the left, depending on the time instant $\left(t_{i}\right)$.

We note that two events may trigger a change in the EEE indicators: (1) a new citation associated to a publication already present in the portfolio and (2) a new publication (with or without citations). Obviously, $E$ does not grow when introducing a new publication with no citations, but this event generally makes $X$ and $S$ vary (see for example the transition between $t_{5}$ and $t_{6}$ ). This is the only case in which some of the EEE indicators may change, with no energy supply from the exterior. In other words, this is the only case of $X$ consumption (and consequent growth of $S$ ) with no increase in $E$. Regarding this behaviour, it is not easy to draw a similarity with thermodynamic systems. What would be the thermodynamic equivalent of a new publication with zero citations? 
Table 3. Chronological sequence of the publication output of a fictitious scientist. Six total publications are considered, reporting their citation accumulation ( $c_{i}$ values in the columns on the left) over time. For each of the time instants $\left(t_{i}\right)$ considered, the following indicators are also reported: $P, C$, $h$, and the three EEE indicators (i.e. $E, X$ and $S$ ).

\begin{tabular}{lrrrrrrrrrrrr}
\hline Instant & $c_{1}$ & $c_{2}$ & $c_{3}$ & $c_{4}$ & $c_{5}$ & $c_{6}$ & $P$ & $C$ & $h$ & $E$ & $X$ & $S$ \\
\hline$t_{1}$ & 0 & 0 & - & - & - & - & 2 & 0 & 0 & 0 & 0.0 & 0.0 \\
$t_{2}$ & 1 & 0 & - & - & - & - & 2 & 1 & 1 & 1 & 0.5 & 0.5 \\
$t_{3}$ & 2 & 0 & - & - & - & - & 2 & 2 & 1 & 4 & 2.0 & 2.0 \\
$t_{4}$ & 2 & 1 & - & - & - & - & 2 & 3 & 1 & 5 & 4.5 & 0.5 \\
$t_{5}$ & 2 & 2 & - & - & - & - & 2 & 4 & 2 & 8 & 8.0 & 0.0 \\
$t_{6}$ & 2 & 2 & 0 & - & - & - & 3 & 4 & 2 & 8 & 5.3 & 2.7 \\
$t_{7}$ & 2 & 2 & 2 & - & - & - & 3 & 6 & 2 & 12 & 12.0 & 0.0 \\
$t_{8}$ & 2 & 2 & 2 & 1 & - & - & 4 & 7 & 2 & 13 & 12.3 & 0.8 \\
$\ldots$ & $\ldots$ & $\ldots$ & $\ldots$ & $\ldots$ & $\ldots$ & $\ldots$ & $\ldots$ & $\ldots$ & $\ldots$ & $\ldots$ & $\ldots$ & $\ldots$ \\
$t_{n}$ & 19 & 14 & 8 & 6 & 3 & - & 5 & 50 & 4 & 666 & 500.0 & 166.0 \\
$t_{n+1}$ & 19 & 14 & 8 & 6 & 3 & 2 & 6 & 52 & 4 & 670 & 450.7 & 219.3 \\
\hline & & & & & & & & & & & &
\end{tabular}

Going on with the comment about Table 3, it is worth remarking that the introduction of a new publication (with the corresponding citations) can never lead to a decrease in $S$. Here follows a demonstration. Table 4 reports (1) the formulae relating to the calculation of $E, X$ and $S$ for a generic set of $P$ publication (instant $t_{P}$ ) and (2) the same formulae in case a new publication with $c_{P+1}$ citations is added (instant $t_{P+1}$ ).

Table 4. Calculation of $E, X$ and $S$ (instant $t_{P}$ ) for a generic set of $P$ publication and (instant $t_{P+1}$ ) in case a new publication with $c_{P+1}$ citations is added.

\begin{tabular}{l|l|l|l|l|l}
\hline Instant & $P$ & $C$ & $E$ & $X$ & $S$ \\
\hline$t_{P}$ & $P$ & $C_{P}=\sum_{i=1}^{P} c_{i}$ & $E_{P}=\sum_{i=1}^{P} c_{i}^{2}$ & $X_{P}=\frac{C_{P}^{2}}{P}$ & $S_{P}=E_{P}-\frac{C_{P}^{2}}{P}$ \\
$t_{P+1}$ & $P+1$ & $C_{P+1}=\sum_{i=1}^{P+1} c_{i}=C_{P}+c_{P+1}$ & $E_{P+1}=E_{P}+c_{P+1}^{2}$ & $X_{P+1}=\frac{C_{P+1}^{2}}{P+1}=\frac{\left(C_{P}+C_{P+1}\right)^{2}}{P+1}$ & $S_{P+1}=E_{P}+c_{P+1}^{2}-\frac{\left(C_{P}+c_{P+1}\right)^{2}}{P+1}$ \\
\hline
\end{tabular}

The variation of $S$ between instants $t_{P}$ and $t_{P+1}$ is given by:

$\Delta S=S_{P+1}-S_{P}=c_{P+1}^{2}-\frac{\left(C_{P}+C_{P+1}\right)^{2}}{P+1}+\frac{C_{P}^{2}}{P}=c_{P+1}^{2}-\frac{P \cdot\left(C_{P}+C_{P+1}\right)^{2}-(P+1) \cdot C_{P}^{2}}{P \cdot(P+1)}$,

which can be turned into this form:

$\Delta S=\frac{\left\{\left[C_{P+1} \cdot P\right]^{2}+C_{P}^{2}-2 \cdot c_{P+1} \cdot P \cdot C_{P}\right\}}{P \cdot(P+1)}=\frac{\left[C_{P}-C_{P+1} \cdot P\right]^{2}}{P \cdot(P+1)}$.

Eq. 14 shows that $\Delta S \geq 0$. The only case in which $\Delta S=0$ is when $C_{P+1}=C_{P} / P$, that is to say, the $\left(c_{P+1}\right)$ citations of the new publication coincide exactly with the average citations per publication of the previous publications $\left(C_{P} / P\right)$. This result is not surprising: in Statistics, the SS of a data set can never decrease when a new entry is added. The only case in which SS does not change is when the new entry is exactly coincident with the mean value of the previous data set (see Eq. 10). 
Prathap underlines that when a new publication is added, "entropy never diminish and can only increase - in consonance with the classical laws of thermodynamics" [Prathap, 2011a, page 522]. This statement is correct but, in Thermodynamics, this applies to isolated systems only, when (spontaneous and irreversible) transformations take place before reaching the equilibrium. Instead, it was shown that biliometric systems are not isolated, therefore this similarity is invoked improperly.

Regarding the bibliometric progress of a scientist, we also note that $S$ can diminish, in those cases in which the "thermodynamically perfect" case (according to Prathap) is approached, i.e. when citations tend to be spread uniformly over the publications. For example, see the transitions between $t_{3}$ and $t_{4}, t_{4}$ and $t_{5}$, and between $t_{6}$ and $t_{7}$, in Table 3 .

The previous Prathap's claim is thus vitiated by the fact that, when he exemplifies the bibliometric progress of a scientist, he adds publications gradually, according to their date of issue, but each publication is associated to the overall "pack" of citations, accumulated up to the moment of the analysis. Table 5 reports one of the examples proposed by Prathap (2011a, page 520). This way, the natural evolution of the bibliometric progress - in particular the gradual accumulation of citations to already issued publications - is not well represented. We think that the representation in Table 3, in which $S$ may also diminish, better reflects the real dynamics of the bibliometric progress of a scientist's publication portfolio.

Table 5. EEE indicators of a scientist's output in chronological sequence. Adapted from (Prathap, 2011a).

\begin{tabular}{crrrrrrrr}
\hline Instant & Date & $P$ & $\Delta c$ & $C$ & $\Delta E$ & $E$ & $X$ & $S$ \\
\hline$t_{1}$ & 1975 & 1 & 0 & 0 & 0 & 0 & 0.00 & 0.00 \\
$t_{2}$ & 1975 & 2 & 2 & 2 & 4 & 4 & 2.00 & 2.00 \\
$t_{3}$ & 1976 & 3 & 5 & 7 & 25 & 29 & 16.33 & 12.67 \\
$t_{4}$ & 1976 & 4 & 13 & 20 & 169 & 198 & 100.00 & 98.00 \\
$t_{5}$ & 1976 & 5 & 14 & 34 & 196 & 394 & 231.20 & 162.80 \\
$t_{6}$ & 1977 & 6 & 0 & 34 & 0 & 394 & 192.67 & 201.33 \\
$t_{7}$ & 1977 & 7 & 6 & 40 & 36 & 430 & 228.57 & 201.43 \\
$t_{8}$ & 1977 & 8 & 6 & 46 & 36 & 466 & 264.50 & 201.50 \\
$t_{9}$ & 1977 & 9 & 7 & 53 & 49 & 515 & 312.11 & 202.89 \\
$t_{10}$ & 1977 & 10 & 9 & 62 & 81 & 596 & 384.40 & 211.60 \\
\hline
\end{tabular}

\section{$4.5 X$ and $p$ non-monotonicity}

The example in Table 3 shows a further curiosity: the introduction of a new publication can sometimes make $X$ decrease. For example, see the transitions between $t_{5}$ and $t_{6}$, and between $t_{n}$ and $t_{n+1}$. This means that $X$, and the $p$-index as well, are non-monotonic performance indicators, which violate the monotonicity property - according to many bibliometrists, a conditio sine qua non for measuring the scientific impact (Woeginger, 2008). By the way, 
examining the definition of $X$ (Eq. 3), it immediately emerges that the introduction of a new publication with no citations leads to a decrease of $X$ ( $C$ does not change, while $P$ increases by one), although this decrease can also occur when the new publications has a positive number of citations. Here is presented an analytical demonstration: let us assume that, in the time instant $t_{P}$, a scientist has $P$ publications with $C$ total citations, while, in the instant $t_{P+1}$, a new publication with $C_{P+1}$ citations is added. This transition may make $X$ decrease, when the following inequality is satisfied:

$$
\Delta X=X_{P+1}-X_{P}=\frac{\left(C_{P}+C_{P+1}\right)^{2}}{P+1}-\frac{C_{P}^{2}}{P} \leq 0 .
$$

The solution of the second-degree inequality is given by:

$$
C_{1}^{*}=-C_{P} \cdot\left(\sqrt{1+\frac{1}{P}}+1\right) \leq c_{P+1} \leq C_{P} \cdot\left(\sqrt{1+\frac{1}{P}}-1\right)=c_{2}^{*} \text {. }
$$

It can be noticed from Eq. 16 that $c_{1}^{*}$ is negative (this condition is always true) and that $c_{2}^{*}$ can be a positive non-integer number. Since $c_{P+1} \in \mathbf{N}_{0}$, Eq. 16 can be reduced to:

$$
c_{P+1} \leq\left\lfloor C_{P} \cdot\left(\sqrt{1+\frac{1}{P}}-1\right)\right\rfloor,
$$

where $\left\lfloor c_{2}^{*}\right\rfloor$ denotes the highest integer not higher than $c_{2}^{*}$. For instance, applying Eq. 17 to the example in Table 3 , at the instant $t_{n}$ (in which $P=5, C_{P}=50$ ), it can be found that $c_{n+1} \leq\lfloor 4.8\rfloor=4$. This means that in the transition between $t_{n}$ and $t_{n+1}$ there can be a reduction in $X$, provided that the new entry publication has no more than 4 publications.

Another inconsistency between the behaviour of thermodynamic and bibliometric systems is given by the fact that it is very difficult to adapt the notions of "spontaneous evolution", "work generation" or "energy transformation" to bibliometric systems.

Also, it is not marginal the fact that bibliometric systems constantly increase their E. Why should they behave like energy accumulators or "black holes"?

\subsection{The notion of thermodynamically perfect case}

It was shown that the "thermodynamically perfect" case is when the number of citations is spread uniformly over the publications of interest (see Eq. 10). The typical evolution of a bibliometric system may (rarely) lead to approach this condition and (more often) to move away from it. A simple interpretation of this tendential, but not necessarily one-way, increase in $S$ is that the distribution of citation counts is usually skewed and the vast majority of 
publications have relatively low citations. The (not very frequent) publications with a relatively high number of citations will therefore make the distribution expand to the right, inducing an increase in the corresponding dispersion [Bornmann et al., 2008; Franceschini and Maisano, 2011b].

Apart from this inconsistency with thermodynamic systems, why should the idea of perfection be associated with the condition of complete uniformity of the citation distribution? Why should the ideal bibliometric "evolutionary processes" tend to such a state? After all, the fact that a scientist's scientific production has some peaks ("big hit" publications) and a tail of low-cited publications is physiological and not necessarily deplorable [Henzinger et al., 2010]. Moreover, we do not understand why - for a given $X$ - a relatively low $S$ should be indicative of "efficiency of scholarly effort". Thus, we think that using the $X / E$ ratio as indicator of efficiency is not very wise, because such indicator would be even more dubious than $X$ or $E$, taken separately. Probably, the classical $C / P$ ratio remains the simplest and most effective indicator for this purpose.

\section{Concluding remarks}

This paper analyses the analogy between the evolution of thermodynamic and bibliometric systems, showing many ambiguities and inconsistencies, which are the inevitable result of some dubious assumptions at the foundations of the model. Overall, numerous explanations and examples given in this article show that:

- $X$ is a dubious performance indicator somehow related to the $h$-index;

- $X$ non-monotonicity is very critical;

- $S$ is an indicator of dispersion improperly invested with the title of indicator of "efficiency and scholarly effort";

- the concepts of iso-energetic and iso-exergetic bibliometric states are not adequately supported;

- the condition of "thermodynamic perfection" is questionable.

Despite these criticisms, we recognize to Prathap the merit of having proposed a fascinating (potential) connection between two worlds (i.e. thermodynamics and bibliometrics) that are apparently so different. Assuming that this analogy can exist, we believe that significant efforts will be needed to reestablish the current model, in order to achieve acceptable consistency. 


\section{References}

Bornmann, L., Mutz, R., \& Daniel, H. D. (2008) Are there better indices for evaluation purposes than the h-index? A comparison of nine different variants of the h-index using data from biomedicine. Journal of the American Society for Information Science and Technology, 59(5), 830-837.

Dincer, I., Cengel, Y.A. (2001) Energy, entropy and exergy concepts and their roles in thermal engineering. Entropy, 3, 116-149.

Franceschini, F., Galetto, M., Maisano, D. (2007) Management by Measurement: Designing Key Indicators and Performance Measurements. Springer: Berlin, ISBN: 9783540732112.

Franceschini, F. \& Maisano, D. (2010) Analysis of the Hirsch index's operational properties. European Journal of Operational Research, 203(2), 494-504.

Franceschini, F., Maisano D. (2011a) Criticism on the hg-index. Scientometrics, 86(2), 339-346.

Franceschini, F., Maisano D. (2011b) Bibliometric positioning of scientific manufacturing journals: a comparative analysis. Scientometrics, 86(2), 463-485.

Franceschini, F. \& Maisano, D. (2011c) Influence of Database Mistakes on Journal Citation Analysis: Remarks on the Paper by Franceschini and Maisano, QREI (2010). To appear in Quality and Reliability Engineering International, DOI: 10.1002/qre.1174.

Glänzel, W. (2006). On the h-index-A mathematical approach to a new measure of publication activity and citation impact. Scientometrics, 67(2), 315-321.

Henzinger M., Sunol J., Weber I. (2010) The stability of the h-index. Scientometrics, 84(2), 465-479.

Hirsch, J.E. (2005). An index to quantify an individual's scientific research output. Proceedings of the National Academy of Sciences of the United States of America, 102, 16569-16572.

Prathap, G. (2010) Is there a place for a mock h-index? Scientometrics, 84(1), 153-165.

Prathap, G. (2011a) The Energy-Exergy-Entropy (or EEE) sequences in bibliometric assessment. Scientometrics, 87(3), 515-524.

Prathap, G. (2011b) A Thermodynamic Explanation for the Glänzel-Shubert Model for the h-Index. Journal of the American Society for Information Science and Technology, 62(5), 992-994.

Schubert, A., Glänzel, W. (2007) A systematic analysis of Hirsch-type indices for journals. Journal of Informetrics, 1(2), 179-184.

Ye, F.Y (2011) A Unification of Three Models for the h-Index. Journal of the American Society for Information Science and Technology, 62(1), 205-207.

Woeginger, G.J. (2008) An axiomatic characterization of the Hirsch-index. Mathematical Social Sciences, 56(2), 224-232. 